01

\title{
Самоуширение и уширение углекислым газом линий молекулы $\mathrm{H}_{2} \mathrm{~S}$
}

\author{
(C) Б.А. Воронин ${ }^{1}$, Н.Н. Лаврентьева ${ }^{1}$, С.С. Воронина ${ }^{1}$, Е.А. Шевченко ${ }^{2}$, А.А. Федорова ${ }^{3}$ \\ ${ }^{1}$ Институт оптики атмосферы им. В.Е. Зуева Сибирского отделения РАН, \\ 643021 Томск, Россия \\ ${ }^{2}$ Томский государственный университет систем управления и радиоэлектроники, \\ 634050 Томск, Россия \\ ${ }^{3}$ Институт космических исследований РАН, \\ 117997 Москва, Россия \\ e-mail: vba@iao.ru, Inn@iao.ru
}

Поступила в редакцию 20.10.2017 г.

В окончательной редакции 15.01.2018 г.

\begin{abstract}
Представлены оценки коэффициентов уширения углекислым газом и самоуширения линий основной изотопической модификации $\mathrm{H}_{2} \mathrm{~S}$, выполненные на основе литературных данных. Проведены оценки $J^{\prime}$ зависимостей указанных параметров контура линий молекулы сернистого водорода. В случае уширения $\mathrm{CO}_{2}$ проведены расчеты полуширин линий полуэмпирическим методом, основанным на параметрической модификации ударной полуклассической модели, параметры модели определены подгонкой к экспериментальным данным.
\end{abstract}

DOI: $10.21883 /$ OS.2018.05.45936.239-17

Сероводород (сернистый водород, сульфид водорода, дигидросульфид) - бинарное химическое соединение водорода и серы, очень токсичен, термически неустойчив (при температурах больше $400^{\circ} \mathrm{C}$ разлагается на простые вещества $-\mathrm{S}$ и $\mathrm{H}_{2}$ ). Молекула сероводорода имеет изогнутую форму, поэтому она полярна (дипольный момент равен 0.974 D). В атмосфере Земли газ является загрязнителем, для него имеются как естественные, так и индустриальные источники, интерес к нему обусловлен необходимостью контроля концентрации этого газа.

С точки зрения планетных исследований наличие в атмосфере короткоживущих соединений серы является одним из косвенных индикаторов недавней геологической активности на планете. По аналогии с Землей вулканическая дегазация на Марсе и Венере должна включать в себя такие серные молекулы, как диоксид серы $\left(\mathrm{SO}_{2}\right)$, сероводород $\left(\mathrm{H}_{2} \mathrm{~S}\right)$ и моноксид серы $(\mathrm{SO})$. Присутствие или отсутствие диоксида серы как основного выделяемого газа является чувствительным индикатором на вулканическую дегазацию на планетах земной группы в современную эпоху [1]. Другие серные соединения, хотя и являются малыми по сравнению c $\mathrm{SO}_{2}$, также могут быть индикатором вулканической активности, и их поиск в атмосферах Венеры и Марса продолжается.

Как на Венере, так и на Марсе основным методом поиска сероводорода является спектроскопия. Используя спектрограф высокого разрешения CSHELL на телескопе HACA IRTF, Краснопольский [2] провел поиск $\mathrm{H}_{2} \mathrm{~S}$, используя линию в области $3.7 \mathrm{mkm}\left(2688.93 \mathrm{~cm}^{-1}\right)$, и нашел верхний предел $23 \mathrm{ppb}$ в пределах $3 \sigma$ на содержание этого газа, что сильно улучшило существующие до этого оценки по наблюдениям в УФ области спектра с верхним пределом $100 \mathrm{ppb}$ [3]. В свою очередь химиче- ская модель [4] предсказывает содержание этого газа на Венере меняющимся от $180 \mathrm{ppb}$ около поверхности до $70 \mathrm{ppb}$ на высоте $47 \mathrm{~km}$. В случае Марса поиск сероводорода был проведен Кхаят и др. [5] на телескопе CSO на Мауна Кеа, Гавайи по линии $300.505 \mathrm{GHz}$ в микроволновом диапазоне. Активных выбросов зарегистрировано не было, но был поставлен верхний предел по уровню $2 \sigma$ по всему диску планеты, равный $1.3 \mathrm{ppb}$. Хотя $\mathrm{H}_{2} \mathrm{~S}$ до сих пор не найден ни на Марсе, ни на Венере, его важность для понимания геологической эволюции этих планет делает интересным уточнение его спектроскопии в углекислых атмосферах для продолжения поиска.

В работе представлены оценки коэффициентов уширения углекислым газом и самоуширения линий основной изотопической модификации $\mathrm{H}_{2}^{32} \mathrm{~S}$, проведенные на основе литературных данных [6-9]. Необходимо отметить, что несмотря на явную зависимость полуширин линий от вращательного квантового числа ( $J^{\prime}$-зависимость), в последних версиях баз данных параметры имеют постоянное значение (в БД HITRAN-2012 [10] уширение воздухом равно $0.074 \mathrm{~cm}^{-1} / \mathrm{atm}$ и самоуширение $-0.158 \mathrm{~cm}^{-1} / \mathrm{atm}$, в БД GEISA-2015 [11] уширение воздухом - $0.08 \mathrm{~cm}^{-1} / \mathrm{atm}$ и самоуширение $\left.0.17 \mathrm{~cm}^{-1} / \mathrm{atm}\right)$. В работе рассматриваются оценки $J^{\prime}$ и $J^{\prime} J^{\prime \prime}$-зависимостей [12] параметров контура линий молекулы сернистого водорода и представлены расчеты $\mathrm{H}_{2} \mathrm{~S}-\mathrm{CO}_{2}$ для 39 переходов.

Когда и кем был введен в обиход термин $J$-зависимость (J-dependent), авторам не известно. Но в [13] термин уже активно используется. Возможно потому, что это достаточно логичный термин, отражающий зависимость параметра контура уширяющего газа (или самоуширения) от вращательного числа $J$. Для большинства молекул данная зависимость является определяющей, но, например, для $\mathrm{SO}_{2}$ влияние вращательного кванто- 
вого числа $K_{a}$ важнее, чем влияние $J$ [14]. Отсутствие индексов ,',"“6 у квантового числа $J$ можно объяснить тем, что в ряде случаев для сокращения записи индекс „'“6, обозначающей принадлежность квантового вращательного числа к нижнему уровню, опускают таким образом, $J=J^{\prime \prime}$. Ранее в работе [12] для водяного пара была установлена зависимость коэффициентов параметра контура не только от вращательного числа $J$, и от того, к какому типу ветви относится переход $P, Q$ или $R$. Это было предложено называть $J^{\prime} J^{\prime \prime}$ зависимостью.

Основное внимание уделено изучению коэффициентов уширения линий $\mathrm{H}_{2} \mathrm{~S}-\mathrm{CO}_{2}$. Кроме оценок на основе литературных данных, проведены расчеты с применением полуэмпирического метода, хорошо зарекомендовавшего себя для ряда сталкивающихся систем и включающего молекулы типа асимметричного волчка в качестве поглощающей компоненты.

\section{Оценки коэффициентов самоуширения линий сернистого водорода}

Самые массовые исследования параметров контура линий сероводорода проведены в работах [6-9]. В работе [7] приведены результаты измерения коэффициентов самоуширения для тринадцати линий полосы $2 v_{2}$ и двух линий полосы $v_{2}$ в спектральном диапазоне $1290-2503 \mathrm{~cm}^{-1}\left(4 \leq J^{\prime \prime} \leq 13,1 \leq K_{a}^{\prime \prime} \leq 5\right)$. Измеренные значения коэффициентов самоуширения лежат в диапазоне $0.11 \leq \gamma\left(\mathrm{H}_{2} \mathrm{~S}-\mathrm{H}_{2} \mathrm{~S}\right) \leq 0.18 \mathrm{~cm}^{-1} / \mathrm{atm}$. Была установлена закономерность, заключающаяся в уменьшении параметров самоуширения с увеличением квантового числа $J$. В работе [9] представлены результаты измерений коэффициентов самоуширения 17 линий полосы $v_{1}$ и 9 линий полосы $v_{3}$ молекулы $\mathrm{H}_{2} \mathrm{~S}$. Спектр был зарегистрирован в диапазоне $2472-2764 \mathrm{~cm}^{-1}\left(3 \leq J^{\prime \prime} \leq 17,0 \leq K_{a}^{\prime \prime} \leq 7\right)$. Измеренные значения коэффициентов самоуширения находятся в интервале $0.12 \leq \gamma\left(\mathrm{H}_{2} \mathrm{~S}-\mathrm{H}_{2} \mathrm{~S}\right) \leq 0.22 \mathrm{~cm}^{-1} / \mathrm{atm}$. Статья [6] содержит расширенный список линий полосы $v_{2}$, измерены коэффициенты самоуширения для 20 линий в диапазоне $1172-1234 \mathrm{~cm}^{-1}\left(1 \leq J^{\prime \prime} \leq 8,0 \leq K_{a}^{\prime \prime} \leq 5\right)$. Значения коэффициентов самоуширения варьируют в пределах $0.12 \leq \gamma\left(\mathrm{H}_{2} \mathrm{~S}-\mathrm{H}_{2} \mathrm{~S}\right) \leq 0.18 \mathrm{~cm}^{-1} / \mathrm{atm}$. В основном полуширины определены для наиболее интенсивных линий поглощения с ограничением $\Delta K_{a} \leq 1$ по вращательному квантовому числу $K_{a}$. В работе [15] для трех линий $\mathrm{H}_{2} \mathrm{~S}$ приводятся значения коэффициентов самоуширения, но отсутствие идентификации не дает возможности их использовать.

На рис. 1 представлена зависимость коэффициента самоуширения $\mathrm{H}_{2} \mathrm{~S}$ от квантового числа углового момента $J^{\prime}$ по данным $[6,7,9]$, на рис. $2, a$ и $2, b-$ соответственно $J^{\prime}$ - и $J^{\prime} J^{\prime \prime}$-зависимости, полученные усреднением параметров контура в зависимости от $J^{\prime}$ и от $J^{\prime \prime}$. Приведенные данные показывают явную зависимость от

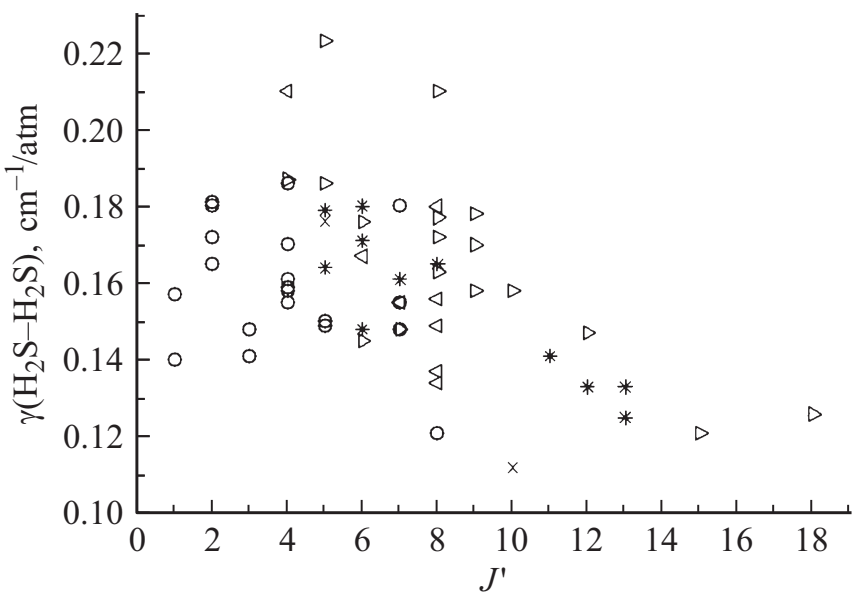

Рис. 1. Коэффициенты самоуширения $\mathrm{H}_{2} \mathrm{~S}$ из $[6,7,9]$ в зависимости от квантового числа $J^{\prime}$ : кружки - [6]; стрелки влево [9], полоса $v_{3}$; стрелки вправо - [9], полоса $v_{1}$; звездочки - [7], полоса $2 v_{2}$; крестики - [7], полоса $v_{2}$.
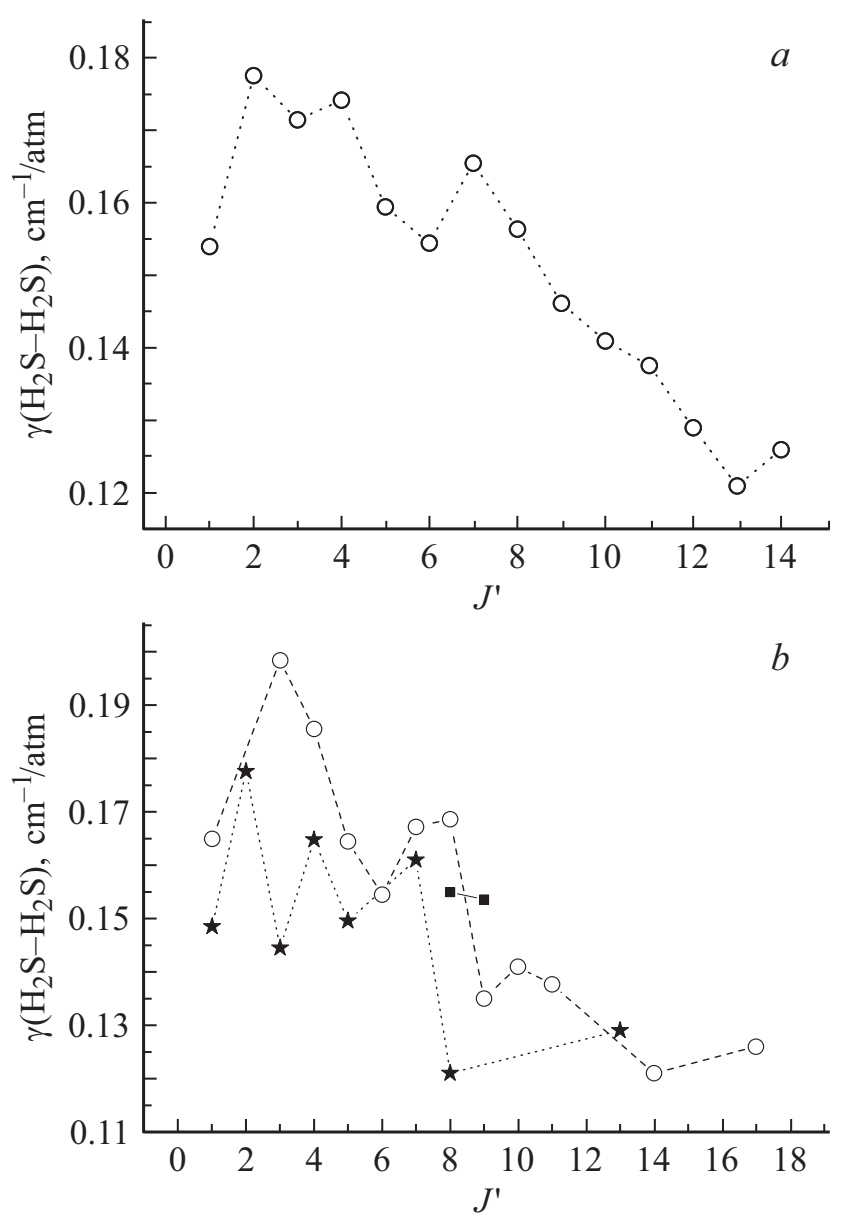

Рис. 2. $a-J^{\prime}$-зависимость коэффициентов самоуширения $\gamma\left(\mathrm{H}_{2} \mathrm{~S}-\mathrm{H}_{2} \mathrm{~S}\right)$, осреднение для всех $J^{\prime} . b-J^{\prime} J^{\prime \prime}$-зависимость коэффициентов самоуширения $\gamma\left(\mathrm{H}_{2} \mathrm{~S}-\mathrm{H}_{2} \mathrm{~S}\right)$ : квадраты $-P$-ветвь, звездочки $-Q$-ветвь, кружки $-R$-ветвь. 
квантовых чисел $J^{\prime}$, для которой подгонкой получено выражение

$$
\gamma\left(\mathrm{H}_{2} \mathrm{~S}-\mathrm{H}_{2} \mathrm{~S}\right)=0.179 e^{-0.02 J^{\prime}}
$$

Сравнение коэффициентов самоуширения линий для полос $v_{1}, v_{2}, 2 v_{2}$ показывает, что колебательная зависимость параметров незначительна.

\section{Коэффициенты уширения линий сернистого водорода давлением углекислого газа}

В работе [8] представлены экспериментально определенные параметры уширения углекислым газом для 39 линий $\mathrm{H}_{2} \mathrm{~S}$ полосы $v_{2}$ в спектральном диапазоне $1050-1325 \mathrm{~cm}^{-1}\left(2 \leq J^{\prime \prime} \leq 11,0 \leq K_{a}^{\prime \prime} \leq 4\right)$, заявлена малая $(2 \%)$ погрешность. Измеренные значения полуширин линий лежат в диапазоне $0.065 \leq \gamma\left(\mathrm{H}_{2} \mathrm{~S}-\mathrm{H}_{2} \mathrm{~S}\right) \leq 0.135 \mathrm{~cm}^{-1} /$ atm. Ha pис. 3 представлена $J^{\prime}$-зависимость величин $\gamma\left(\mathrm{H}_{2} \mathrm{~S}-\mathrm{CO}_{2}\right)$ по цитированным данным. Она хорошо аппроксимируется выражением

$$
\gamma\left(\mathrm{H}_{2} \mathrm{~S}-\mathrm{CO}_{2}\right)=157.7 e^{-0.07 J^{\prime}} .
$$

Полуширины линий в различных полосах заметно отличаются. Для $Q$-ветви мало данных, поэтому, возможно, имеет смысл параметры $P$ - и $R$-ветвей рассматривать отдельно, используя $J^{\prime} J^{\prime \prime}$-зависимость, а для $Q$-ветви использовать $J^{\prime}$-зависимость.

Чтобы рассчитать коэффициенты уширения спектральных линий молекулы сероводорода давлением $\mathrm{CO}_{2}$, мы использовали полуэмпирический метод. Данный подход работает в рамках следующих приближений ударной теории: рассматриваются бинарные столкновения, относительное движение взаимодействующих молекул описывается классическими траекториями, продолжительность столкновений меньше, чем время между

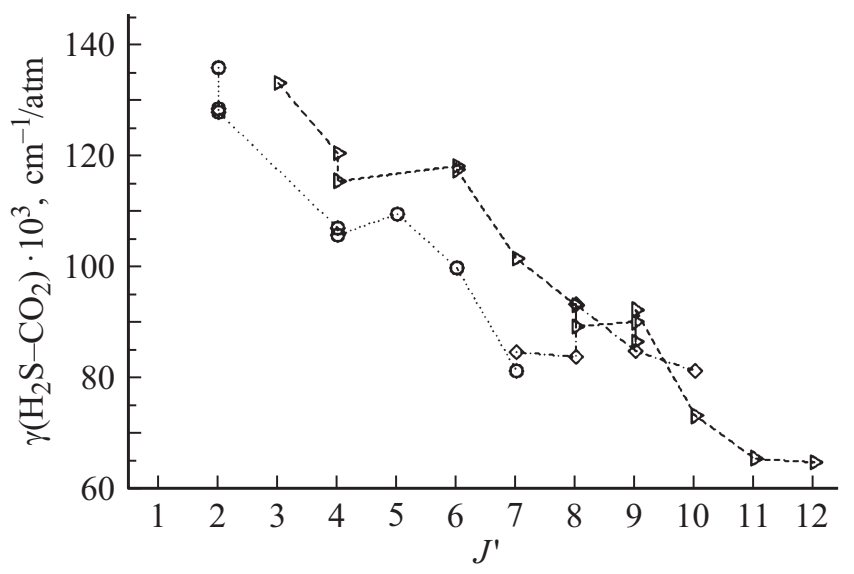

Рис. 3. Коэффициенты уширения $\gamma\left(\mathrm{H}_{2} \mathrm{~S}-\mathrm{CO}_{2}\right)$ в зависимости от углового момента $J^{\prime}$ по данным [8]: кружки $-P$-ветвь, ромбы $-Q$-ветвь, треугольники $-R$-ветвь. столкновениями, интерференция линий не принимается во внимание.

При таких допущениях полуширина линии $\gamma_{i f}$, соответствующая переходу $i \rightarrow f$, записывается как

$$
\gamma_{f i}+i \delta_{f i}=\frac{n}{c} \sum_{p} \rho(p) \int_{0}^{\infty} v f(v) d v \int_{0}^{\infty} b U(i, f, p, b, v) d b .
$$

Реальная часть функции эффективности $U(i, f, p, b, v)$ представляется следующим образом:

$$
\begin{aligned}
& \operatorname{Re} U(i, f, p, b, v)=1-\left\{1-S_{2, f p . i p}^{(L)}\right\} \\
& \times \cos \left[S_{1, f p}-S_{1, i p}+\operatorname{Im} S_{2, f p}-\operatorname{Im} S_{2, i p}\right] \\
& \quad \times \exp \left[-\left(\operatorname{Re} S_{2, f p}+\operatorname{Re} S_{2, i p}+S_{2, f p, i p}^{(C)}\right)\right],
\end{aligned}
$$

где $\rho(p)$ - заселенность уровня $p, n-$ плотность возмущающих частиц, $p$ - набор квантовых чисел второй (возмущающей) молекулы, $v-$ относительная скорость сталкивающихся молекул, $f(v)$ - функция распределения по скоростям Максвелла, $b$ - прицельное расстояние, $S_{1}$ и $S_{2}$ - выражения первого и второго порядка по теории возмущений, индексы $(L)$ и $(C)$ соответствуют „связным“ (linked) и „присоединенным“ (connected) диаграммам теории возмущений. Член первого порядка $S_{1}$ отвечает за адиабатический эффект и определяется только изотропной частью потенциала, а $S_{2}$ - его анизотропной частью. Обе функции зависят от „классической“ траектории относительного движения сталкивающихся молекул.

Выражения (3) и (4) зависят от сил дипольных переходов $D^{2}\left(i i^{\prime} \mid l\right)$ и $D^{2}\left(f f^{\prime} \mid l\right)$ различных каналов рассеяния $i \rightarrow i^{\prime}, f \rightarrow f^{\prime}$, связывающих нижние и верхние уровни перехода с другими близко расположенными уровнями. Эти параметры являются квадратом редуцированных матричных элементов молекулярных постоянных, таких как дипольный момент или компоненты квадрупольного тензора.

Полуширина линии в теории Андерсона выражаются следующим образом:

$$
\begin{aligned}
\gamma_{i f}= & A(i, f)+\sum_{i^{\prime}} D^{2}\left(i i^{\prime} \mid l\right) P_{l}\left(\omega_{i i^{\prime}}\right) \\
& +\sum_{f^{\prime}} D^{2}\left(f f^{\prime} \mid l\right) P_{l}\left(\omega_{f f^{\prime}}+\ldots\right),
\end{aligned}
$$

где

$$
\begin{aligned}
P_{l}(\omega)= & \frac{n}{c} \sum_{p} \rho(p) \sum_{l^{\prime}, p^{\prime}} A_{l l^{\prime}} D^{2}\left(p p^{\prime} \mid l^{\prime}\right) F_{l l^{\prime}} \\
& \times\left(\frac{2 \pi c b_{0}(p, i, f)}{v}\left(\omega+\omega_{p p^{\prime}}\right)\right)
\end{aligned}
$$

- функция эффективности каналов рассеяния $i \rightarrow i^{\prime}$ или $f \rightarrow f^{\prime}\left(\omega_{i i^{\prime}}\right.$ или $\omega_{f f^{\prime}}$ обозначены как $\left.\omega\right)$. Слагаемое теории Андерсона, обусловленное прерыванием, 
Экспериментальные данные [8] и настоящий расчет

\begin{tabular}{|c|c|c|c|c|c|c|c|c|c|c|}
\hline \multirow[t]{2}{*}{ Ветвь } & \multirow[t]{2}{*}{$v_{0}, \mathrm{~cm}^{-1}$} & $J^{\prime}$ & $K_{a}^{\prime}$ & $K_{c}^{\prime}$ & $J$ & $K_{a}$ & $K_{c}$ & $\begin{array}{c}\gamma\left(\mathrm{H}_{2} \mathrm{~S}-\mathrm{CO}_{2}\right) \cdot 10^{3} \\
\mathrm{~cm}^{-1} / \mathrm{atm}\end{array}$ & $\begin{array}{c}\gamma\left(\mathrm{H}_{2} \mathrm{~S}-\mathrm{CO}_{2}\right) \cdot 10^{3} \\
\mathrm{~cm}^{-1} / \mathrm{atm}\end{array}$ & \multirow[t]{2}{*}{$\begin{array}{c}{ }^{* *} \text { Сравнение эксперимента } \\
\text { и расчета, \% }\end{array}$} \\
\hline & & \multicolumn{3}{|c|}{ верхние } & \multicolumn{3}{|c|}{ нижние } & {$[8]$} & настоящий расчет & \\
\hline$P$ & 1149.7695 & 2 & 1 & 2 & 3 & 0 & 3 & $128.6 \pm 1.5$ & 133.3 & -3.65 \\
\hline$P$ & 1149.4014 & 2 & 0 & 2 & 3 & 1 & 3 & $135.8 \pm 1.5$ & 134.8 & 0.74 \\
\hline$P$ & 1144.2649 & 2 & 2 & 1 & 3 & 1 & 2 & $127.8 \pm 0.8$ & 129 & -0.94 \\
\hline$P$ & 1117.4890 & 4 & 3 & 2 & 5 & 2 & 3 & $107.0 \pm 0.6$ & 112.6 & -5.23 \\
\hline$P$ & 1122.8520 & 4 & 1 & 3 & 5 & 2 & 4 & $105.9 \pm 0.7$ & 106 & -0.09 \\
\hline$P$ & 1105.7183 & 5 & 2 & 3 & 6 & 3 & 4 & $109.7 \pm 1.1$ & 108.1 & 1.46 \\
\hline$P$ & $1110.9888^{*}$ & 6 & 0 & 6 & 7 & 1 & 7 & $100.0 \pm 2.0$ & 82.8 & 17.20 \\
\hline$P$ & $1110.9890^{*}$ & 6 & 1 & 6 & 7 & 0 & 7 & $100.0 \pm 2.0$ & 82.8 & 17.20 \\
\hline$P$ & 1089.5537 & 7 & 2 & 5 & 8 & 3 & 6 & - & 86.5 & \\
\hline$P$ & 1089.6241 & 7 & 3 & 5 & 8 & 2 & 6 & - & 86.2 & \\
\hline$P$ & $1095.6985^{*}$ & 7 & 1 & 6 & 8 & 2 & 7 & - & 81.3 & \\
\hline$P$ & $1095.6958^{*}$ & 7 & 2 & 6 & 8 & 1 & 7 & - & 81.2 & \\
\hline$P$ & $1101.0584^{*}$ & 7 & 1 & 7 & 8 & 0 & 8 & $81.4 \pm 0.6$ & 80.2 & 1.47 \\
\hline$P$ & $1101.0584^{*}$ & 7 & 0 & 7 & 8 & 1 & 8 & $81.4 \pm 0.6$ & 80.2 & 1.47 \\
\hline$R$ & 1263.4153 & 3 & 3 & 1 & 2 & 0 & 2 & $133.1 \pm 1.4$ & 124.7 & 6.31 \\
\hline$R$ & 1253.4644 & 4 & 3 & 2 & 3 & 2 & 1 & $120.5 \pm 0.5$ & 123.6 & -2.57 \\
\hline$R$ & 1266.9334 & 4 & 4 & 1 & 3 & 3 & 0 & $115.6 \pm 0.7$ & 105.2 & 9.00 \\
\hline$R$ & 1304.0056 & 5 & 3 & 2 & 4 & 2 & 3 & - & 121.8 & \\
\hline$R$ & 1322.5491 & 6 & 4 & 2 & 5 & 3 & 3 & $118.2 \pm 0.6$ & 120 & -1.52 \\
\hline$R$ & 1286.5657 & 6 & 4 & 3 & 5 & 3 & 2 & - & 121.4 & \\
\hline$R$ & 1303.7024 & 6 & 5 & 2 & 5 & 4 & 1 & $117.5 \pm 0.6$ & 117.2 & 0.26 \\
\hline$R$ & 1279.7048 & 7 & 2 & 5 & 6 & 3 & 4 & $101.6 \pm 0.4$ & 95.2 & 6.30 \\
\hline$R$ & 1289.8918 & 8 & 3 & 6 & 7 & 2 & 5 & $93.2 \pm 0.4$ & 87.1 & 6.55 \\
\hline$R$ & 1289.8357 & 8 & 2 & 6 & 7 & 3 & 5 & $89.5 \pm 0.5$ & 86.9 & 2.91 \\
\hline$R$ & 1299.7693 & 9 & 3 & 7 & 8 & 2 & 6 & $90.2 \pm 2.8$ & 82.2 & 8.87 \\
\hline$R$ & 1299.7585 & 9 & 2 & 7 & 8 & 3 & 6 & $86.7 \pm 1.3$ & 82.1 & 5.31 \\
\hline$R$ & 1313.2622 & 9 & 3 & 6 & 8 & 4 & 5 & $92.5 \pm 0.7$ & 88.4 & 4.43 \\
\hline$R$ & $1294.5083^{*}$ & 10 & 1 & 9 & 9 & 2 & 8 & $73.4 \pm 0.5$ & 79.9 & -8.86 \\
\hline$R$ & $1294.5083^{*}$ & 10 & 2 & 9 & 9 & 1 & 8 & $73.4 \pm 0.5$ & 79.9 & -8.86 \\
\hline$R$ & 1286.8428 & 11 & 1 & 11 & 10 & 0 & 10 & $65.8 \pm 0.4$ & 66.9 & -1.67 \\
\hline$R$ & $1295.0949^{*}$ & 12 & 0 & 12 & 11 & 1 & 11 & $65.1 \pm 0.4$ & 66.3 & -1.84 \\
\hline$R$ & $1295.0949^{*}$ & 12 & 1 & 12 & 11 & 0 & 11 & $65.1 \pm 0.4$ & 66.2 & -1.69 \\
\hline$Q$ & 1276.0224 & 7 & 2 & 6 & 7 & 1 & 6 & $84.9 \pm 0.3$ & 81.9 & 3.53 \\
\hline$Q$ & 1252.8633 & 8 & 2 & 6 & 8 & 1 & 7 & $84.0 \pm 3.0$ & 81.7 & 2.74 \\
\hline$Q$ & 1252.8745 & 8 & 3 & 6 & 8 & 2 & 7 & $93.4 \pm 1.1$ & 81.6 & 12.63 \\
\hline$\tilde{Q}$ & $1263.0420^{*}$ & 9 & 2 & 7 & 9 & 1 & 8 & $85.1 \pm 0.6$ & 80.3 & 5.64 \\
\hline$Q$ & $1263.0442^{*}$ & 9 & 3 & 7 & 9 & 2 & 8 & $85.1 \pm 0.6$ & 80.3 & 5.64 \\
\hline$Q$ & $1273.0593^{*}$ & 10 & 2 & 8 & 10 & 1 & 9 & $81.4 \pm 0.4$ & 80.0 & 1.72 \\
\hline$Q$ & $1273.0598^{*}$ & 10 & 3 & 8 & 10 & 2 & 9 & $81.4 \pm 0.4$ & 80.0 & 1.72 \\
\hline
\end{tabular}

Примечание.

* Сильно перекрывающиеся линии.

** $\left(\left(\gamma_{\text {эксп }}-\gamma_{\text {расч }}\right) / \gamma_{\text {эксп }}\right) 100$.

где $b_{0}(v, p, i, f)$ - параметр прерывания, представляет собой выражение

$$
A(i, f)=\frac{n}{c} \sum_{p} \rho(p) \int_{0}^{\infty} v F(v) b_{0}^{2}(v, p, i, f) d v .
$$

В (6) $l=1$ соответствует дипольным, а $l=2-$ квадрупольным переходам в поглощающей молекуле. Вероятности переходов $D^{2}\left(i i^{\prime} \mid l\right)$ и $D^{2}\left(f f^{\prime} \mid l\right)$ относятся к каналам рассеивания $i \rightarrow i^{\prime}, f \rightarrow f^{\prime}$ и зависят только от свойств поглощающей молекулы, в то время как функ- ция эффективности $P_{l}\left(\omega_{i i^{\prime}}\right)$ зависит от межмолекулярного потенциала, траектории относительного движения молекул, уровней энергии и волновых функций буферной молекулы. В работе [16] было предложено функцию эффективности представить следующим образом:

$$
P_{l}(\omega)=P_{l}^{A} C_{l}(\omega),
$$

где $P_{l}^{A}(\omega)-$ функция эффективности в теории Андерсона. Здесь $C_{l}(\omega)$ - корректирующий фактор, вид которого может быть определен из рассмотрения зависимости от вращательных квантовых чисел, а параметры из 


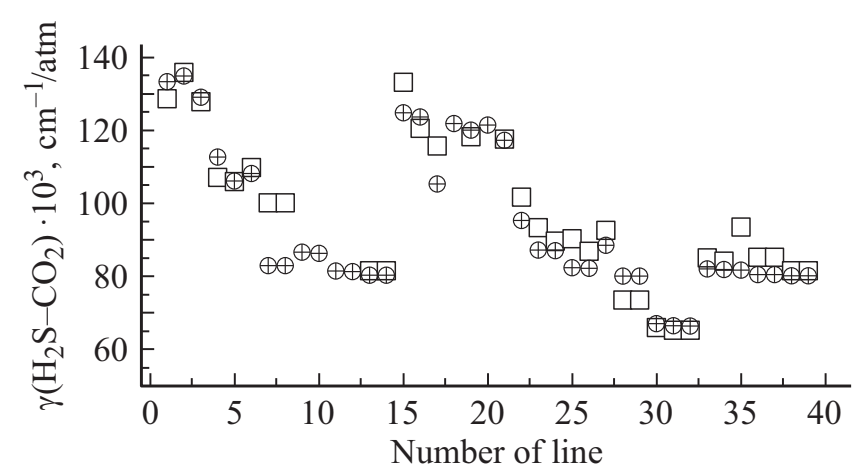

Рис. 4. На рисунке представлены данные из таблицы. Нумерация переходов согласно таблице. Перекрещенными кружками представлен наш расчет, квадратами - эксперимент [8].

подгонки к экспериментальным значениям полуширин линий.

В рассматриваемом случае соотношение (8) записывалось в виде

$$
P_{l}\left(\omega_{f f^{\prime}}\right)=P_{l}^{A}\left(\omega_{f f^{\prime}}\right)\left[c_{1} /\left(c_{2} \sqrt{j_{f}}+1\right)\right] .
$$

Были получены следующие параметры подгонки: $c_{1}=1.276$ и $c_{2}=0.075$. В рассмотрение были включены диполь-дипольное, диполь-квадрупольное, квадрупольквадрупольное взаимодействия, а также индукционный и дисперсионный члены поляризационного потенциала. Главный вклад в уширение линии обусловлен взаимодействием между дипольным моментом сероводорода и квадрупольным моментом углекислого газа.

В таблице представлены измеренные и рассчитанные коэффициенты уширения линий $\mathrm{H}_{2} \mathrm{~S}$, величины варьируют от $73 \cdot 10^{-3}$ до $135 \cdot 10^{-3} \mathrm{~cm}^{-1} / \mathrm{atm}$.

О согласии расчетных и экспериментальных данных можно судить по данным из таблицы и рис. 4. Максимальное отклонение расчета от эксперимента составляет $17.2 \%$ (имеет место для сильно перекрывающихся линий), минимальное - $0.09 \%$, среднее отклонение $2.6 \%$. На сайте ftp://ftp.iao.ru/pub/VTT/H2S-CO2/ выложены файлы в формате баз данных БД HITRAN и GEISA-15 с параметрами контура линий $\mathrm{H}_{2} \mathrm{~S}$, названия файлов: „HITRAN-2016-H2S-all_Iso-g_co2.par“ и „line_GEISA 2003_asc_gs03_v1.0_h2s-gCO2-gself“. В БД GEISA-15 самоуширение линии для молекулы $\mathrm{H}_{2} \mathrm{~S}$ одинаково для всех переходов $\left(0.17 \mathrm{~cm}^{-1} \mathrm{~atm}\right)$, в нашем файле постоянное значение заменено на $J$-зависимое, коэффициенты уширения воздухом заменены на коэффициенты уширения $\mathrm{H}_{2} \mathrm{~S}-\mathrm{CO}_{2}$. В остальном данные файлы оставлены без изменения (ftp://ftp.iao.ru/pub/VTT/H2S-CO2/ line_GEISA2003_asc_gs03_v1.0_h2s-gCO2-gself). Изменения в файле выполнены для всех изотопологов. Аналогичная процедура проведена и для HITRAN-16 c той лишь разницей, что самоуширение по сравнение с HITRAN-12 уже изменено с константы на более точные зависимости, поменяли только уширение воздухом на уширение $\mathrm{CO}_{2}$ для всех изотопологов в файле ftp://ftp.iao.ru/pub/VTT/H2S-CO2/ HITRAN-2016-H2S-all_Iso-g_co2.par , в остальном coхранен оригинальный формат. Файл readmeH2S-CO2.txt содержит описание директории.

Работа выполнена при поддержке комплексной программы фундаментальных научных исследований Сибирского отделения РАН № II.2П „Интеграция и развитие“ ІІ.2П/II.10-29, „Спектроскопическое обеспечение исследований химического состава атмосфер и климата планет“ № 0368-2015-0002. Часть работы, касающаяся самоуширения линий сероводорода, поддержана грантом РФФИ № 17-52-16022. Кроме того, исследование проведено в рамках проекта АААА-А17-117021310147-0.

\section{Список литературы}

[1] Andres R.J., Rose W.I., Kyle P.R., de Silva S., Francis P., Gardeweg M., Roa H.M. // J. Volc. Geotherm. Res. 1991. V. 46. P. 323. doi 10.1016/0377-0273(91)90091-d

[2] Krasnopolsky V.A. // Icarus. 2008. V. 197. P. 377. doi 10.1016/j.icarus.2008.05.020

[3] Owen T., Sagan C. // Icarus. 1972. V. 16. P. 557.

[4] Krasnopolsky V.A. //Icarus. 2007. V. 191. P. 25.

[5] Khayat A.S., Villanueva G.L., Mumma M.J., Tokunaga A.T. // Icarus. 2015. V. 253. P. 130. doi 10.1016/j.icarus.2015.02.028.

[6] Waschull J., Kuhnemann F., Sumpf B. // J. Mol. Spectrosc. 1994. V. 165. P. 150.

[7] Sumpf B. // J. Mol. Spectrosc. 1997. V. 181. P. 160.

[8] Kissel A., Sumpf B., Kronfeldt H.-D., Tikhomirov B.A., Ponomarev Yu.N. // J. Mol. Spectrosc. 2002. V. 216. P. 345. doi 10.1006/jmsp.2002.8630

[9] Sumpf B., Meusel I., Kronfeldt H.-D. // J. Mol. Spectroscopy. 1996. V. 177. P. 143.

[10] Rothman L.S., Gordon I.E., Babikov Y., Barbe A., Benner D.C., Bernath P.F., Birk M., Bizzocchi L., Boudon V., Brown L.R., Campargue A., Chance K., Coudert L., Devi V.M., Drouin B.J., Fayt A., Flaud J.-M., Gamache R.R., Harrison J., Hartmann J.-M., Hill C., Hodges J.T., Jacquemart D., Jolly A., Lamouroux J., LeRoy R.J., Li G., Longo D., Mackie C.J., Massie S.T., Mikhailenko S., Muller H.S.P., Naumenko O.V., Nikitin A.V., Orphal J., Perevalov V., Perrin A., Polovtseva E.R., Richard C., Smith M.A.H., Starikova E., Sung K., Tashkun S., Tennyson J., Toon G.C., Tyuterev Vl.G., Vander Auwera J., Wagner G. // JQSRT. 2013. V. 130. P. 4.

[11] Jacquinet-Husson N., Armante R., Scott N.A., Chédin A., Crépeau L., Boutammine C., Bouhdaoui A., Crevoisier C., Capelle V., Boonne C., Poulet-Crovisier N., Barbe A., Chris Benner D., Boudon V., Brown L.R., Buldyreva J., Campargue A., Coudert L.H., Devi V.M., Down M.J., Drouin B.J., Fayt A., Fittschen C., Flaud J.-M., Gamache R.R., Harrison J.J., Hill C., Hodnebrog O., Hu S.-M., Jacquemart D., Jolly A., Jiménez E., Lavrentieva N.N., Liu A.W., Lodi L., Lyulin O.M., Massie S.T., Mikhailenko S., Müller H.S.P., Naumenko O.V., Nikitin A., Nielsen C.J., Orphal J., Perevalov V.I., Perrin A., Polovtseva E., PredoiCross A., Rotger M., Ruth A.A., Yu S.S., Sung K., Tashkun S.A., Tennyson J., Tyuterev Vl.G., Vander Auwera J., Voronin B.A., Makie A. // J. Mol. Spectrosc. 2016. V. 327. P. 31. 
[12] Voronin B.A., Lavrentieva N.N., Mishina T.P., Chesnokova T.Yu., Barber M.J., Tennyson J. // JQSRT. 2010. V. 111. P. 2308.

[13] Rahn L.A., Palmer R.E. // JOSA B. 1986. V. 3. P. 1164-1169.

[14] Voronin B.A., Kasatkin S.S. // Proc. SPIE. 2015. V. 9680. P. $1-5$. doi 10.1117/12.2205503

[15] Ciaffoni L., Cummings B.L., Denzer Peverall R., Procter S.R., Ritchie G.A.D. // Appl. Phys. B. 2008. V. 92. P. 627.

[16] Bykov A., Lavrentieva N., Sinitsa L. // Mol. Phys. 2004. V. 102. P. 1706. 